\title{
VIDEO SEGMENTATION FOR MOVING OBJECT DETECTION USING LOCAL CHANGE \& ENTROPY BASED ADAPTIVE WINDOW THRESHOLDING
}

\author{
Anuradha.S.G ${ }^{1}$, Dr.K.Karibasappa ${ }^{2}$, Dr.B.Eswar Reddy ${ }^{3}$ \\ ${ }^{1}$ Department of Computer Science \& Engineering, RYMEC, Bellary, Karnataka, \\ India. \\ anuradha_gagadinerediffmail.com \\ ${ }^{2}$ Department of Information Science \& Engineering, DSCE, Bangalore, India. \\ K_karibasappa@hotmail.com \\ ${ }^{3}$ Department of Computer Science \& Engineering, \\ JNTUA, Anantapur, Andhra Pradesh, India \\ eswarcsejntu@gmail.com
}

\begin{abstract}
Motion detection and object segmentation are an important research area of image-video processing and computer vision. The technique and mathematical modeling used to detect and segment region of interest (ROI) objects comprise the algorithmic modules of various high-level techniques in video analysis, object extraction, classification, and recognition. The detection of moving object is significant in many tasks, such as video surveillance \& moving object tracking. The design of a video surveillance system is directed on involuntary identification of events of interest, especially on tracking and on classification of moving objects. An entropy based realtime adaptive non-parametric window thresholding algorithm for change detection is anticipated in this research. Based on the approximation of the value of scatter of sections of change in a difference image, a threshold of every image block is calculated discriminatively using entropy structure, and then the global threshold is attained by averaging all thresholds for image blocks of the frame. The block threshold is calculated contrarily for regions of change and background. Investigational results show the proposed thresholding algorithm accomplishes well for change detection with high efficiency.
\end{abstract}

\section{KEYWORDS}

Video Segmentation, Object Detection, Object Tracking, Video Surveillance, motion detection.

\section{INTRODUCTION}

The development of video databases has impelled research for structuring multimedia content. Traditionally, low-level descriptions are provided by image and video segmentation techniques.

Natarajan Meghanathan et al. (Eds) : ITCSE, ICDIP, ICAIT - 2013

pp. 155-166, 2013. () CS \& IT-CSCP 2013

DOI : $10.5121 /$ csit.2013.3916 
The best segmentation is achieved by the human eye, performing simultaneously segmentation and recognition of the object thanks to a strong prior knowledge about the objects' structures. To generate similar high-level descriptions, a knowledge representation should be used in computerbased systems. One of the challenges is to map efficiently the low-level descriptions with the knowledge representation to improve both segmentation and interpretation of the scene.

There are three key steps in video analysis: detection of interesting moving objects, tracking of such objects from frame to frame, and analysis of object tracks to recognize their behaviour.

In its simplest form, Segmentation of moving objects in image sequences is one of the key issues in computer vision, since it lies at the base of virtually any scene analysis problem. In particular, segmentation of moving objects is a crucial factor in content- based applications such as interactive TV, content-based scalability for video coding, content-based indexing and retrieval, etc. Obviously, such applications require an accurate and stable partition of an image sequence to semantically meaningful objects.

\section{PROBlem \& Related WORK}

Commonly, two approaches exist for detecting moving objects in a sequence of images. The first approach relies on change detection as the source of temporal information. These approaches are motivated by the assumption that moving objects usually entail intensity changes between successive frames. Hence, moving objects can be identified by applying a decision rule on the intensity differences between successive frames in a sequence. Various decision rules have been suggested to identify moving objects. Yet, these approaches suffer from two major drawbacks. First, unless moving objects are sufficiently textured, only occlusion areas (covered background) will be marked as changed, while the interior of objects will remain unchanged.

Second, uncovered background will be marked as changed in the process as well, thus the boundaries of the moving objects are likely to be inaccurate. To overcome this drawback, a postprocessing step that distinguishes between moving objects and uncovered background has to be applied.

The aim of an object tracker is to generate the trajectory of an object over time by locating its position in every frame of the video. Object tracker may also provide the complete region in the image that is occupied by the object at every time instant. The tasks of detecting the object and establishing correspondence between the object instances across frames can either be performed separately or jointly.

Depending on the tracking domain, a tracker can also provide object-centric information, such as orientation, area, or shape of an object. One can simplify tracking by imposing constraints on the motion and/or appearance of objects.

The basic framework of moving object detection for video surveillance is shown in figure below. 


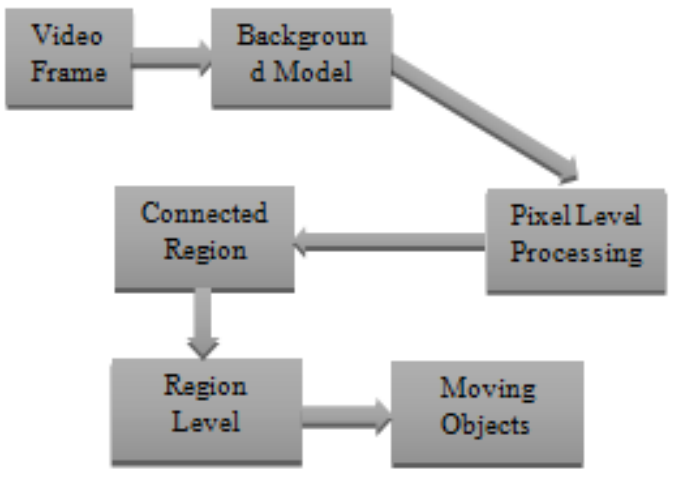

Figure1. Framework for Basic Video Object Detection System

In computer vision and video processing areas, moving object detection is a very important research topic. The process of moving object detection in video consists of two steps background extraction and moving object detection. The preliminary idea is to capture a series of video pictures at regular intervals; the video is divided into $n$ number of frames to describe the vector information of the region.Rajaguru et al. in [40], develops a work in Moving object detection using draw pad, experiments show that detected objects in real streaming video is converted into drawing shapes or figures in a specified area. A paper [41] begins with a discussion of the deficiency of current algorithms which use the number of the changing points of frame-difference as the threshold to determine a moving object.

A number of techniques and algorithms have been developed for the object segmentation task in the past [1-5]. The technique presented in certain researches segments moving objects directly relied on motion information. The affine parametric clustering approach provided a layered description of video by its motion similarity depicted in [1]. The technique in [2] combined motion estimation and segmentation within the Bayesian framework. The interdependence between the optical flow estimation and the segmentation map has been reinforced iteratively. In [3], an integrated Bayesian approach was proposed to extract motion layers. Yet, most physical objects usually exhibit non-rigid motion and cannot be characterized by a parametric model, thus motion-based moving object segmentation techniques result in a finer partition than actually requirement.

In contrast with the motion-based approach, region-based object segmentation technique is developed to accommodate the accurate segmentation of objects [4-5]. The spatial partition of frame is firstly performed to obtain homogeneous regions. The location of object boundary is guided by the initial spatial segmentation. Therefore, the extracted objects can succeed in object boundaries that are not clearly distinct. In [4], the first frame of a video sequence is partitioned into homogeneous regions based on intensity. A region merging process grouped the regions into object based on motion similarity. In order to get meaningful spatial partition, human interaction and the morphological segmentation tool was combined to obtain a complete and accurate extraction of video object in the first frame [5].

Level set methods are also important in problems with current scenario, in a research [42], firstly, an improved frame difference algorithm is used for dealing with the problem of initial moving objective detection in video sequence, with adjacent video frame subtracting technique, moving 
objective pixels in current image can be detected by adaptive threshold. Then expanding level set $\mathrm{C}-\mathrm{V}$ model, the contours of moving object should be extracted with the energy function evolving. An efficient Modified Directional Lifting-based 9/7 Discrete Wavelet Transform (MDLDWT) structure is proposed in [43] to further reduce the computational cost and preserve the fine shape information in low resolution image.

The most challenging part of moving objects detection in moving background is that motion induced by camera moving may dominate the observed motion, which makes existing methods cannot detect the real moving objects from the moving background robustly and computationally efficient. Xie, Songhua et al in [44], proposed a very fast method that can detect moving objects from moving background accurately without prior knowledge of the camera motion characteristics. Also, a Multi-layer Codebook-based Background Subtraction (MCBS) model is proposed in [45] for video sequences to detect moving objects.

Turbulence mitigation refers to the stabilization of videos with non uniform deformations due to the influence of optical turbulence. Typical approaches for turbulence mitigation follow averaging or dewarping techniques. Although these methods can reduce the turbulence, they distort the independently moving objects, which can often be of great interest, [46] address the novel problem of simultaneous turbulence mitigation and moving object detection.

Moving object detection is an imperative constituent of a traffic surveillance structure. Usual background subtraction methodologies often poorly achieve on a long out-of-doors traffic video due to vehicles waiting at an intersection \& gradual variations of illumination and background shadow location. [47] present a fast and vigorous background subtraction algorithm established on unified spatio-temporal background and foreground demonstration. The connection between neighbouring pixels provides high levels of detection accurateness in the dynamic background scene. Author's method (Bayesian fusion), which establishes the traffic flow scene model, associates both background \& foreground models and deliberates prior probabilities to adapt changes of background in every frame.

There has been a increasing research curiosity in video image segmentation over the past period $\&$ towards this end, a wide variability of procedures have been established [18]-[21].

The video segmentation methodologies have extensively used stochastic image models, particularly Markov Random Field (MRF) model, as the model for video sequences [22]-[24]. MRF model has proved to be an effective stochastic model for image segmentation [25]-[27] because of its attribute to model context dependent entities such as image pixels and correlated features. In Video segmentation, besides spatial modelling and constraints, temporal constraints are also added to devise spatio-temporal image segmentation schemes.

An adaptive clustering algorithm has been reported [32] where temporal constraints and temporal local density have been adopted for smooth transition of segmentation from frame to frame. Spatio-temporal segmentation has also been applied to image sequences [28] with different filtering techniques. Extraction of moving object and tracking of the same has been achieved in spatio-temporal framework [29] with Genetic algorithm serving as the optimization tool for image segmentation.

Recently, MRF model has been used to model spatial entities in each frame [29] and Distributed Genetic algorithm (DGA) has been used to obtain segmentation. Modified version of DGA has 
been proposed [23] to obtain segmentation of video sequences in spatio-temporal framework. Besides, video segmentation and foreground subtraction has been achieved using the spatiotemporal notion [30]-[31] where the spatial model is the Gibbs Markov Random Field and the temporal changes are modelled by mixture of Gaussian distributions. Very recently, automatic segmentation algorithm of foreground objects in video sequence segmentation has been proposed [32].

\section{The PROPOSED AlgorithM}

The proposed algorithm computes a threshold of each block of an image adaptively based on the scatter of regions of change (ROC) using the local change adaptive thresholding and averages all thresholds for image blocks to obtain the global threshold based on entropy values. To enhance the segmentation results, the results thus obtained from ROC thresholding section are verified and compensated by considering the information of the pixels belonging to objects in the previous frame. After deciding the window size entropy of the window is computed from the gray level distribution of the window and background subtraction (non ROC region) is done after calculation of entropy.

Entropy-based methods consequence in algorithms that use the entropy of the foreground and background sections, the cross-entropy between the original and binarized image, etc. The entropy of a system as defined by Shannon gives a measure of our ignorance about its actual structure.

One of the primary disturbances sources are uneven lighting, which often exists in the capturing of an image, especially during field operation. The main causes for uneven lighting are:

1. The light may not be always stable

2. The object is so large such that it creates an uneven distribution of the light, and

3. The background is unable to be optically isolated from shadows of other objects.

One possible solution to this problem is to partition the whole image into certain small windows, and then use those existing methods to threshold each small window. This process is called thresholding in partitioned windows. The smaller the window size is, the better the result will be. However, when the window size becomes too small, it can produce the problem of homogeneous windows, i,e., windows contain only background or object pixels. As a consequence, black areas called ghost objects will occur after thresholding. Therefore, there is a need to develop a new technique for automatically selecting window size in order to obtain optimal result i.e., adaptive window selection. This technique is based on the pyramid data structure manipulation, and the window size is adaptively selected according to Shannon's entropy measure.

If a global thresholding algorithm is applied in a difference image, affected by noise, illumination variation or shading:

i. Few pixels that actually correspond to the background in an image frame are identified as changed pixels, whereas these are actually not.

ii. It also happens that a pixel in the difference image that correspond to actual change region and lies in the lower range of the histogram may be identified as an unchanged pixel. 
An adaptive thresholding approach can be used to overcome these problems. However the choice of window size is an issue.

In this regards we propose entropy based adaptive window selection scheme to determine the block/ window size in background segmentation process. Here the threshold value for a particular window is obtained by Otsu's thresholding scheme. To enhance the segmentation results, the results thus obtained from ROC segmentation are verified and compensated by considering the information of the pixels belonging to objects in the previous frame. This is represented as

$$
R=\left\{r_{s} \rightarrow 0 \leq s \leq(A-1)(B-1)\right\}
$$

Where, $R$ is a matrix having the same size of the frame, $s$ is the element number in the matrix and $r_{s}$ is the value of the VOP at location $s$. If a pixel is found to have $r_{s}=1$, it is a part of the moving object of the previous frame; otherwise it belongs to the background of the previous frame.

Based on this information, Thresholding in Background (Non ROC) is modified as follows: if it belongs to a moving object part in the previous frame and its label obtained by temporal segmentation is the same as one of the corresponding pixels in the previous frame, the pixel is marked as the foreground area in the current frame else as a background.

\section{A. Thresholding In ROC}

First, the output ${ }^{D_{n}}$ of change detection at time instant $\mathrm{n}$, is divided into $\mathrm{K}$ equal-sized blocks. Then a ROC scatter estimation process (Described below) is applied, where each image block $W_{k}$, $\mathrm{k}=\{1,2, \cdots, \mathrm{K}\}$, is marked either as containing ROC, denoted $W_{k}^{r}$, or not containing ROC, denoted $W_{k}^{b}$. The threshold $T_{k}^{b}$ of a $W_{k}^{b}$ is computed by a noise statistical-testing algorithm. The threshold $T_{k}^{r}$ of a $W_{k}^{r}$ is computed by a noise-robust thresholding method. That is, the threshold $T_{k}$ of a $W_{k}$ in $D_{n}$ is defined as

$$
T_{k}=\left\{\begin{array}{l}
T_{k}^{r} \text { of } W_{k}^{\gamma} \\
T_{k}^{b} \text { of } W_{k}^{b}
\end{array}\right.
$$

Finally, the global threshold $T_{n}$ of a difference image $D_{\text {nis }}$

$$
T_{n}=\frac{1}{K} \sum_{k=1}^{K} T_{k}
$$

Since size and velocity of objects, noise, local changes in videos may affect the histogram of a $\mathrm{w}_{\mathrm{k}}$, the first moment of histogram is used to estimate the scatter of ROC making the estimation (Eq.4 and 5) adaptive to these characteristics. We also account for local changes (Eq. 6).

The ROC in $D_{n}$ are, in general, scattered over the $K$ image blocks. Let ${ }^{i}$ be a pixel in $D_{n}$ that varies between 0 and 255. i is high in ROC which are caused by strong changes such as motion or significant illumination changes and is low in non ROC which are caused by slight changes such as noise or slight illumination changes. We use the first moment, $\mathrm{m}_{\mathrm{k}}$, of the histogram of each image block $\mathrm{w}_{\mathrm{k}}$ as a measure for determining if an image block contains ROC. 


$$
m_{k}=\sum_{i=1}^{g_{m \Delta x}} i \cdot F_{i}
$$

Where $F_{i}$ is the frequency of Gray-level $\mathrm{i}=\left\{1,2, \cdots, \mathrm{g}_{\max }\right\}$ and $\mathrm{g}_{\max }$ is the maximum Gray-level in the image block. If ${ }^{m_{k}}$ of $w_{k}$ is greater than a threshold ${ }^{T_{m}}$, the image block is regarded as a block

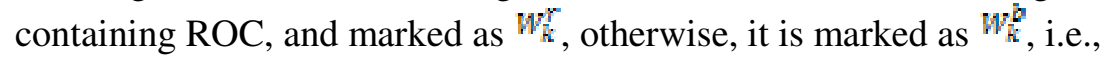

$$
w_{k}=\left\{\begin{array}{l}
w_{k}^{b} m_{k} \leq T_{m} \\
w_{k}^{r} m_{k}>T_{m}
\end{array}\right.
$$

$m_{\vec{h}}$ May vary greatly under different video conditions. We propose, therefore, to adaptively determine $T_{\mathrm{m}}$ as follows. Non-zero pixels in image blocks without ROC are in general caused by noise, illumination changes, or background movement. Those factors usually affect an image globally thus $\mathrm{m}_{\mathrm{k}}$ for different $w_{k}^{k}$ should be similar to each other. To find ${ }^{T_{\mathrm{m}}}$, we first compute the $\mathrm{m}_{\mathrm{k}}$ of each block and then descending sort them $\mathrm{k}$ values. A straight line between the first bin and the last filled bin is then drawn. ${ }^{T_{m i}}$ s selected to maximize the perpendicular distance between the line and the sorted first moment curve.

For each image block $w_{k}^{\pi}$ containing ROC, we propose the local-change adaptive thresholding. First, the histogram of each $W_{k}^{\pi}$ is computed and divided into L equal partitions, and the most frequent gray-level $g_{f l}, l=\{1,2, \cdots, \mathrm{L}\}$, in each histogram partition is fixed. Then the average Gray level $\mu_{k}$ of $W_{k}^{r}$ is computed and the threshold $T_{k}^{\gamma}$ of $W_{k}^{r}$ is obtained by averaging the sum of all $\theta_{f l}$ and $\mu_{k}$, i.e.,

$$
T_{k}^{r}=\frac{\sum_{t=2}^{+} g_{f 1}+\mu_{k}}{L+1}
$$

\section{B. Thresholding in background (Non ROC)}

The basic notion of window growing is to fix the window size primarily focussing on the information measure of the image at different scale. In other words, fixing the size of the window not only depends on the entropy of the chosen window but also the feature entropy of the window. The edges of the window are considered as the features and the feature entropy is computed. Since, the edge map represents the image information at a different scale, the entropy at this scale also plays a pivotal role for image segmentation. Thus, the basic notion is to capture the information at a different scale. In this approach an arbitrarily small window (here the window size $w$ is chosen as $5 \times 5$ ) is considered initially and the entropy of the window is computed from the gray level distribution of the window and is denoted by $\mathrm{H}_{\mathrm{w}}$

$$
H_{w}=\sum_{i=1}^{G} p_{i} \log _{s}\left(\frac{1}{p_{i}}\right)
$$

Where $p_{i}$ the probability distribution of the $\mathrm{i}^{\text {th }}$ Gray value is, $H_{w}$ denotes entropy of the window, $\mathrm{G}$ denotes the total number of Gray values. Over a given window, the edge map is computed and the entropy of the edge map is

$$
H_{w f}=\sum_{i=1}^{G} p_{f i} \log _{e}\left(\frac{1}{p_{n}}\right)
$$


Where ${ }^{H_{w i f}}$ denotes the entropy of the edge map of the window. The following are the two window growing criterion.

If the entropy of the window is comparable to some fraction of the entropy of the whole image, (represented as $T h$ ), that window is chosen for segmentation (by Otsu's thresholding); otherwise the window will be incremented by $\Delta w$ (here $\Delta w$ is considered 2) and the condition will be tested again. The window will be fixed if the total image is exhausted. The final threshold image is obtained by taking union of each considered threshold windows.

The window is fixed if the following is satisfied

$$
H_{w}>T h
$$

Where Th is selected based on the entropy of the total image.

The following criterion is considered for window fixing after grow of the window.

$$
H_{w}>T h
$$

Subject to the constraint,

$$
H_{w f}>T h_{f}
$$

The thresholds Th and ${ }^{T h_{f}}$ for the above inequality are chosen based on the total entropy of the image and that of edge map respectively. Empirically, it is found that the thresholds are closer to the entropy of the whole image and whole edge map. First a window of size ${ }^{m \times n}$ is chosen and it is merged with size ${ }^{a \times b}$ to make a window of size ${ }^{(m+a) \times(n+b)}$ and so on till condition is not satisfied. After fixing of one window, another of size $m \times n$ started from adjacent side. The followings are the steps of the algorithm.

\section{RESULTS}

We have modified the thresholding step of this algorithm since often leads to a significant level of miss classifications.

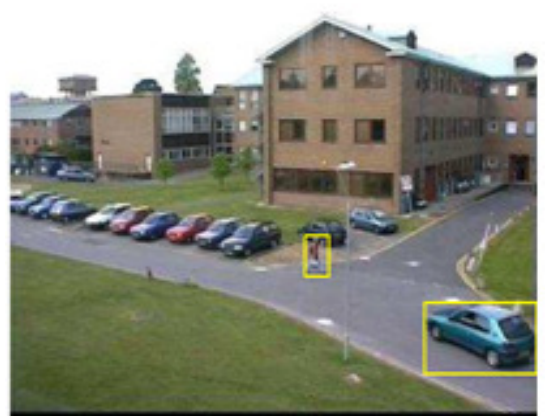

Figure 2 Two regions (in bounding boxes) of an image 


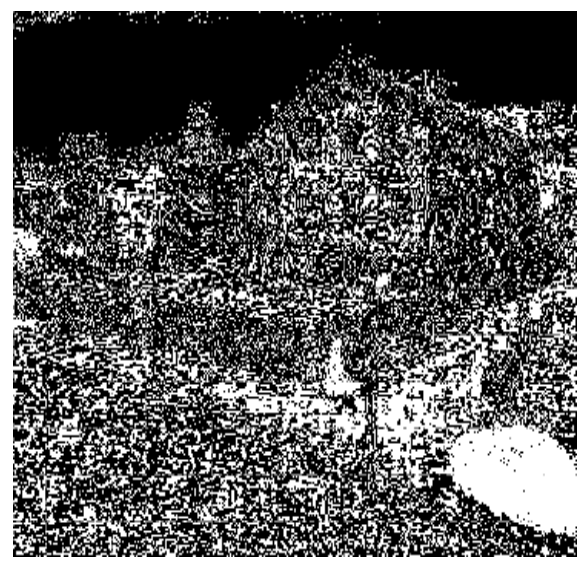

Figure 3 Initial Adaptive Thresholding on interconnected basis for the original input frame

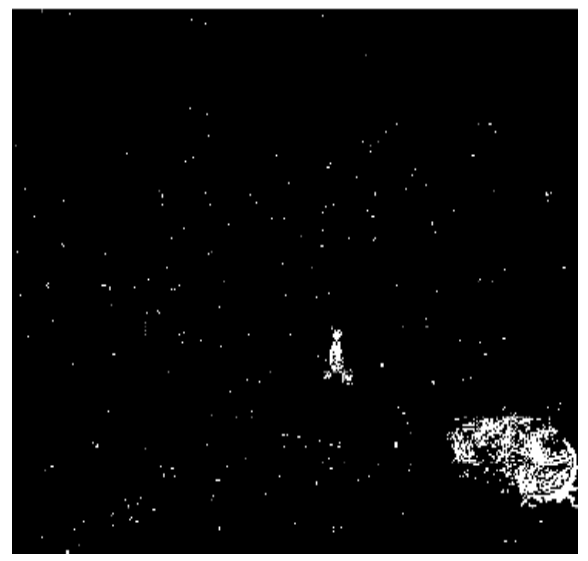

Figure $4 \mathrm{~T}_{\mathrm{m}}$ is selected to maximize the perpendicular distance between the line and the sorted first moment curve

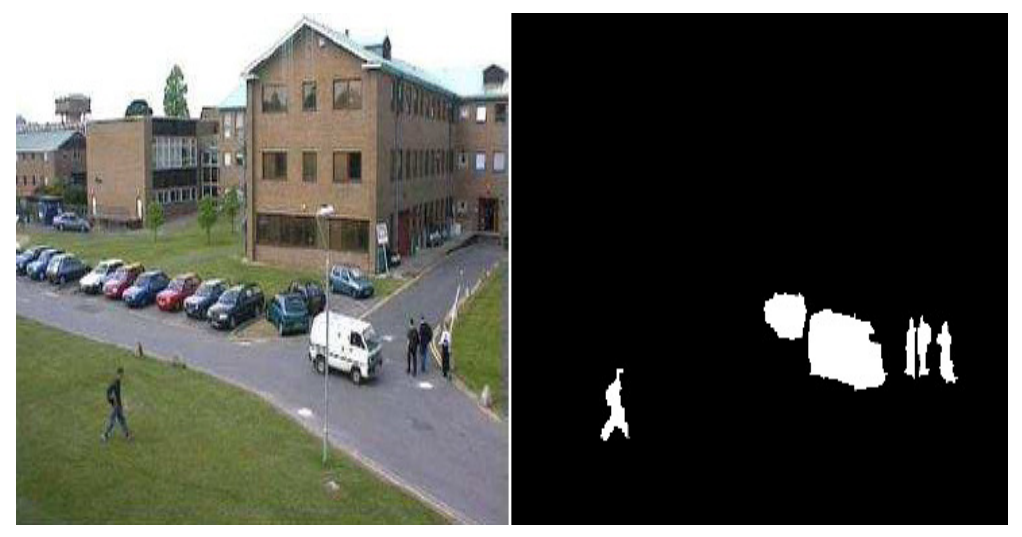

Figure 5. Input frame and segmented image by the user 


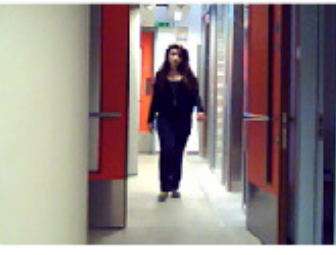

(a)

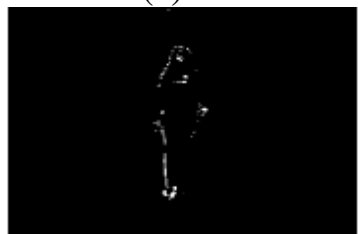

(c)

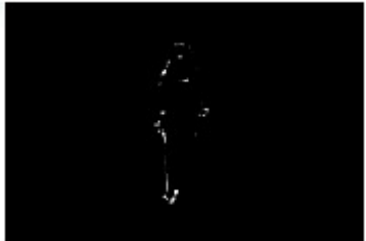

(b)

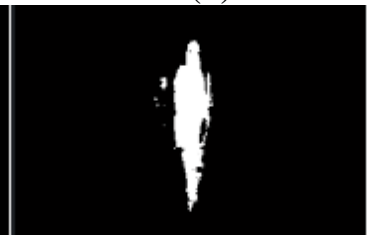

(d)

Figure 6. (a) Shows the original frame content in a video stream, (b) and (c) shows foreground abstraction through adaptive thresholding and window based thresholding (d) shows final segmentation through entropy based threshold selection

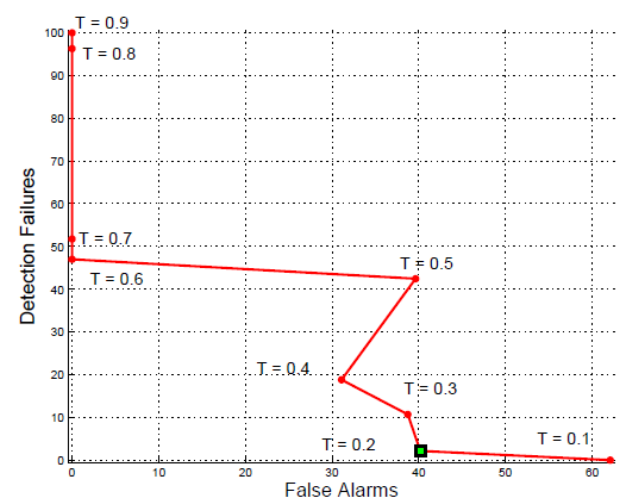

Figure 7. Detection failure characteristics for different false alarm for input streams

\section{CONCLUSIONS}

Real-time entropy based adaptive window thresholding method for video object change detection is proposed in this research. Local changes are taken into account. Based on the estimation of the scatter of regions of change of a difference image, a threshold of each image block is computed discriminatively with the help of entropy scheme. The universal threshold is the average of all thresholds of image blocks. Both the visual assessment and the objective estimation show that the proposed method clearly outperforms the other traditional methods and is steadier than the spatial properties based methods. The efficiency of the proposed method is much sophisticated than the spatial properties based methods. 


\section{REFERENCES}

[1] M.M.Chane. kM.Tekalo. andM.I.Sezan. "Simultaneous motion estimation and segmentation," IEEE Transaction on image Processing, VoL6, No. 9, pp. 1326-1333, Sept. 1997.

[2] J.Wangand E.Adelson, " Representing moving images with laver." IEEE Transaction on Image Processing. Vo1.3. NoS.DD. 6i5-638, Sept. 1994.

[3] P.H.S.Torr, RSzeliski, and P.Anandan,"An integrated Bayesian approach to layer extraction from image sequence," IEEE Transaction on Pattern Analysis and Machine Intelligence, Vol. 23, No.3, pp. 297-303, Mar. 2001.

[4] D. Wang, "Unsupervised video segmentation based on watersheds and temporal tracking," IEEE Transaction on Circuits and Systems for Video Technology, Vol. 8, No.5, Sept. 1998

[5] C.Gu and M.C.Lee, "Semiautomatic segmentation and tracking of semantic video objects,"IEEE Transaction on Circuits and Systems for Video Technology, Vo1.8, No.5, Sept 1998.

[6] Hiraiwa, A., Accurate estimation of optical flow for fully automated tracking of moving-objects within video streams, Circuits and Systems, 1999. ISCAS '99. Proceedings of the 1999 IEEE International Symposium

[7] Takaya, K., Tracking a video object with the active contour (snake) predicted by the optical flow, Electrical and Computer Engineering, 2008. CCECE 2008. Canadian Conference

[8] Jayabalan, E., Non Rigid Object Tracking in Aerial Videos by Combined Snake and Optical flow Technique, Computer Graphics, Imaging and Visualisation, 2007. CGIV '07

[9] Bauer, N.J., Object focused simultaneous estimation of optical flow and state dynamics, Intelligent Sensors, Sensor Networks and Information Processing, 2008. ISSNIP 2008. International Conference

[10] Ping Gao, Moving object detection based on kirsch operator combined with Optical Flow, Image Analysis and Signal Processing (IASP), 2010 International Conference

[11] Pathirana, P.N., Simultaneous estimation of optical flow and object state: A modified approach to optical flow calculation, Networking, Sensing and Control, 2007 IEEE International Conference

[12] Tiesheng Wang, Moving Object Tracking from Videos Based on Enhanced Space-Time-Range Mean Shift and Motion Consistency, Multimedia and Expo, 2007 IEEE International Conference

[13] Khan, Z.H., Joint particle filters and multi-mode anisotropic mean shift for robust tracking of video objects with partitioned areas, Image Processing (ICIP), 2009 16th IEEE International Conference

[14] YuanyuanJia, Real-time integrated multi-object detection and tracking in video sequences using detection and mean shift based particle filters, Web Society (SWS), 2010 IEEE 2nd Symposium

[15] Sung-Mo Park, Object tracking in MPEG compressed video using mean-shift algorithm, Information, Communications and Signal Processing, 2003 and Fourth Pacific Rim Conference on Multimedia. Proceedings of the 2003 Joint Conference of the Fourth International Conference

[16] Khan, Z.H., Joint anisotropic mean shift and consensus point feature correspondences for object tracking in video, Multimedia and Expo, 2009. ICME 2009. IEEE International Conference

[17] Yilmaz, A., Object Tracking by Asymmetric Kernel Mean Shift with Automatic Scale and Orientation Selection, Computer Vision and Pattern Recognition, 2007. CVPR '07. IEEE Conference

[18] A. M. Teklap, Digital Video Processing. Prentice Hall, NJ, 1995.

[19] P. Salember and F. Marques, "Region based representation of image and video segmentation tools for multimedia services,"IEEE Trans. Circuit systems and video Technology, vol. 9, No. 8, pp. 11471169, Dec.1999.

[20] E. Y. Kim, S. H. Park and H. J. Kim, "A Genetic Algorithm-based segmentation of Random Field Modeled images," IEEE Signal processing letters, vol. 7, No. 11, pp. 301-303, Nov. 2000.

[21] S. Geman and D. Geman, "Stochastic relaxation, Gibbs distributions and the Bayesian restoration of images," IEEE Trans. on Pattern Analysis and Machine Intelligence, vol. 6, No. 6, pp. 721-741, Nov. 1984.

[22] R. O. Hinds and T. N. Pappas, "An Adaptive Clustering algorithm for Segmentation of Video Sequences," Proc. of International Conference on Acoustics, speech and signal Processing, ICASSP, vol. 4, pp. 2427-2430, May. 1995.

[23] E. Y. Kim and K. Jung, "Genetic Algorithms for video Segmentation," Pattern Recognition, vol. 38, No. 1, pp.59-73, 2005. 
[24] E. Y. Kim and S. H. Park, "Automatic video Segmentation using genetic algorithms," Pattern Recognition Letters, vol. 27, No. 11, pp. 1252-1265, Aug. 2006.

[25] Stan Z. Li, Markov field modeling in image analysis. Springer: Japan, 2001.

[26] J. Besag, "on the statistical analysis of dirty pictures," Journal of Royal Statistical Society Series B (Methodological), vol. 48, No. 3, pp.259-302, 1986.

[27] A. L. Bovic, Image and Video Processing. Academic Press, New York, 2000.

[28] G. K. Wu and T. R. Reed, "Image sequence processing using spatiotemporal segmentation," IEEE Trans. on circuits and systems for video Technology, vol. 9, No. 5, pp. 798-807, Aug. 1999.

[29] S. W. Hwang, E. Y. Kim, S. H. Park and H. J. Kim, "Object Extraction and Tracking using Genetic Algorithms," Proc. of International Conference on Image Processing, Thessaloniki, Greece, vol.2, pp. 383-386, Oct. 2001.

[30] S. D. Babacan and T. N. Pappas, "Spatiotemporal algorithm for joint video segmentation and foreground detection," Proc. EUSIPCO, Florence, Italy, Sep. 2006.

[31] S. D. Babacan and T. N. Pappas, "Spatiotemporal Algorithm for Background Subtraction," Proc. of IEEE International Conf. on Acoustics, Speech, and Signal Processing, ICASSP 07, Hawaii, USA, pp. 1065-1068, April 2007.

[32] S. S. Huang and L. Fu," Region-level motion-based background modeling and subtraction using MRFs," IEEE Transactions on image Pocessing, vol.16, No. 5, pp.1446-1456, May. 2007.

[33] Comaniciu, D., Meer, P., "Mean shift: A robust approach toward feature space analysis", IEEE Transactions on Pattern Analysis and Machine Intelligence, vol. 24, no. 5, pp. 603-619, 2002.

[34] Shan, C., Tan, T., Wei, Y., "Real-time hand tracking using a mean shift embedded particle filter", Pattern Recognition, Vol. 40, No. 7, pp. 1958-1970, 2007.

[35] Yilmaz, A., Javed, O., Shah, M., "Object tracking: A survey", ACM Computing Surveys, Vol. 38, No. 4, Article No. 13 (Dec. 2006), 45 pages, doi: 10.1145/1177352.1177355, 2006.

[36] Comaniciu, D., Meer, P., "Mean Shift Analysis and Applications", IEEE International Conference Computer Vision (ICCV'99), Kerkyra, Greece, pp. 1197-1203, 1999.

[37] Kass, M., Witkin, A., and Terzopoulos, D., "Snakes: active contour models", International Journal of Computer Vision, Vol. 1, No. 4, pp. 321-331, 1988.

[38] T. Bernier, J.-A. Landry, "A New Method for Representing and Matching Shapes of Natural Objects", Pattern Recognition 36, 2003, pp. 1711-1723.

[39] H. Blum, "A Transformation for extracting new descriptors of Shape", in: W. Whaten-Dunn(Ed.), MIT Press, Cambridge, 1967. pp. 362-380.

[40] Rajaguru, D., Moving object detection using draw pad, Advances in Engineering, Science and Management (ICAESM), 2012 International Conference

[41] HaihuiGao, A New Detection Algorithm of Moving Objects Based on Human Morphology, Intelligent Information Hiding and Multimedia Signal Processing (IIH-MSP), 2012 Eighth International Conference

[42] Rongguo Zhang, Moving Objective Detection and Its Contours Extraction Using Level Set Method, Control Engineering and Communication Technology (ICCECT), 2012 International Conference

[43] Chih-Hsien Hsia, Improved directional lifting-based discrete wavelet transform for low resolution moving object detection, Image Processing (ICIP), 2012 19th IEEE International Conference

[44] Xie, Songyun, Fast detecting moving objects in moving background using ORB feature matching, Intelligent Control and Information Processing (ICICIP), 2013 Fourth International Conference

[45] Juo, J., Fast Background Subtraction Based on a Multi-Layer CodeBook Model for Moving Object Detection, Circuits and Systems for Video Technology, IEEE Transactions 